\title{
Alterations of RNA splicing patterns in esophagus squamous cell carcinoma
}

\author{
Jiyu Ding ${ }^{1,2}$, Chunquan Li $i^{3}$, Yinwei Cheng ${ }^{1,2}$, Zepeng Du ${ }^{4}$, Qiuyu Wang ${ }^{3}$, Zhidong Tang ${ }^{3}$, Chao Song 3 , \\ Qiaoxi Xia ${ }^{1,2}$, Wenjing Bai ${ }^{1,2}$, Ling Lin ${ }^{1,2}$, Wei Liu ${ }^{1,2}$, Liyan $X u^{1,5}$, Enmin $\mathrm{Li}^{1,2^{*}}$ and Bingli Wu $\mathrm{W}^{1,2^{*}}$ (1)
}

\begin{abstract}
Alternative splicing (AS) is an important biological process for regulating the expression of various isoforms from a single gene and thus to promote proteome diversity. In this study, RNA-seq data from 15 pairs of matched esophageal squamous cell carcinoma (ESCC) and normal tissue samples as well as two cell lines were analyzed. AS events with significant differences were identified between ESCC and matched normal tissues, which were re-annotated to find protein coding genes or non-coding RNAs. A total of 45,439 AS events were found. Of these, 6019 (13.25\%) significant differentially AS events were identified. Exon skipping (SE) events occupied the largest proportion of abnormal splicing events. Fifteen differential splicing events with the same trends of $\triangle \Psi$ values in ESCC tissues, as well in the two cell lines were found. Four pathways and 20 biological processes related to pro-metastasis cell junction and migration were significantly enriched for the differentially spliced genes. The upregulated splicing factor SF3B4, which regulates 92 gene splicing events, could be a potential prognostic factor of ESCC. Differentially spliced genes, including HNRNPC, VCL, ZNF207, KIAA1217, TPM1 and CALD1 are shown with a sashimi plot. These results suggest that cell junction- and migration-related biological processes are influenced by AS abnormalities, and aberrant splicing events can be affected by splicing factor expression changes. The involved splicing factor SF3B4 was found to be a survivalrelated gene in ESCC and is presumed to regulate AS in multiple cancers. In summary, we identified significant differentially expressed AS events which may be related to the development of ESCC.
\end{abstract}

Keywords: Alternative splicing, MISO, Esophagus squamous cell carcinoma

\section{Introduction}

Esophageal cancer is the sixth leading cause of cancer fatalities in the world [1], with a five-year survival of less than $20 \%$ [2]. Esophageal cancer is mainly classified into two types that differ in epidemiology and pathology: esophageal squamous cell carcinoma (ESCC) and esophageal adenocarcinoma (EAC). About $90 \%$ of esophageal cancer cases worldwide are ESCC [1]. Studies committed to the molecular mechanism of ESCC development need

*Correspondence: nmli@stu.edu.cn; blwu@stu.edu.cn

${ }^{2}$ Department of Biochemistry and Molecular Biology, Shantou University Medical College, Shantou 515041, China

Full list of author information is available at the end of the article to be carried out further to help improve the diagnosis, treatment and prognosis of this detrimental disease.

Pre-mRNA splicing is a biological process, carried out by a spliceosome consisting of five snRNP complexes and more than 200 auxiliary proteins [4], that removes intron lariats and joins exons together to produce mature mRNAs [3]. In the process of alternative splicing, alternative splice site selection within pre-mRNAs results in the production of various mature mRNAs from a single gene [5]. It has been reported that alternative splicing plays important roles in normal organ development and cell differentiation [6]. However, splicing pattern abnormalities caused by mutations of cis-acting sequences and spliceosomal proteins can also trigger human diseases, including cancer [7, 8]. Altered splicing

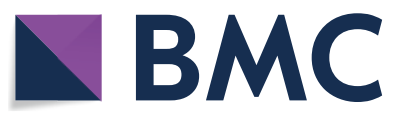

(c) The Author(s) 2021. This article is licensed under a Creative Commons Attribution 4.0 International License, which permits use, sharing, adaptation, distribution and reproduction in any medium or format, as long as you give appropriate credit to the original author(s) and the source, provide a link to the Creative Commons licence, and indicate if changes were made. The images or other third party material in this article are included in the article's Creative Commons licence, unless indicated otherwise in a credit line to the material. If material is not included in the article's Creative Commons licence and your intended use is not permitted by statutory regulation or exceeds the permitted use, you will need to obtain permission directly from the copyright holder. To view a copy of this licence, visit http://creativeco mmons.org/licenses/by/4.0/. The Creative Commons Public Domain Dedication waiver (http://creativecommons.org/publicdomain/ zero/1.0/) applies to the data made available in this article, unless otherwise stated in a credit line to the data. 
patterns influence multiple aspects of cancer development including cell proliferation, programmed cell death, metabolism, angiogenesis, and tumor metastasis [5, 9]. Genome-wide studies indicate that tumor development often involves large-scale variations in alternative splicing [10].

Recent developments in high-throughput sequencing technologies and computational tools have revolutionized our capacity to investigate AS at the genome-wide level. In 2018, changes in exon usage were found across the full TCGA cohort, where tumor samples had an increase in abnormal splicing events as compared to normal samples for the majority of the investigated cancers [11]. Using PSI (percent spliced in) and DEXSeq methods, 28 pairs of colorectal cancer and normal tissues were analyzed, where 9 significant AS genes were found with two splicing events associated with patient overall survival [12]. Applying public data from TCGA, more than 200 DEGs (Differentially Expressed Genes) with AS events were identified and a risk score based on 12 genes associated with overall survival was established in colon adenocarcinoma[13]. In kidney renal clear cell carcinoma, 13 survival-specific genes were significantly correlated with AS events from SpliceSeq database [14].

The MISO model was originally developed to assess the expression of alternatively spliced exons and transcripts [15]. MISO uses confidence intervals to assess exon and transcript abundance, but also detects differentiallyspliced exons or transcripts. This is accomplished by calculating the posterior distribution of unobserved random variables from the RNA-Seq data [15]. The combination of RNA-seq data and computational tools enables AS analyses at a genome-wide level, which provides insight into AS mechanisms and potential clinical application. In this study, MISO was utilized to characterize the splicing patterns and try to identify the regulatory roles of splicing factors on splicing abnormalities in ESCC.

\section{Materials and methods}

\section{Source of sequencing data and routine analysis}

RNA-seq data of 15 pairs of ESCC tissues was generated from our laboratory, which has been published in the previous study [16]. FastQC was used to measure the quality of the sequences [17]. The paired-end sequences were then aligned against the UCSC (http://genome.ucsc.edu/) human genome (hg19) [18] using TopHat2 v2.0.13 [19] and Bowtie2 [20] using default parameters. Alignments were further analyzed with Cufflinks [21] to calculate the abundance (FPKM) of each gene and isoform using the hg19 gene annotations, and with DESeq [22] to identify differentially-expressed splicing factors. To validate the differentially-expressed splicing factors, two microarray datasets, named GSE53624 [23] and GSE23400 [24], were utilized to perform differential expression analysis with the siggenes package in R2.15.3. The RNA-seq data from a pair cell lines, an immortalized human esophageal epithelial cell line (SHEE) and its malignant transformed cell line (SHEEC) [16], was applied as the test cohort in this study.

\section{Identification of AS events in ESCC}

The splicing patterns of ESCC were analyzed with MISO [15] using exon-centric annotations V.2.0, and the results were filtered with a cut-off of $\Delta \Psi>0.2$, Bayes-factor $>5$, and a sum of inclusion and exclusion reads number $>10$. Sashimi plots were used to visualize AS events with significant differences which occurred in $\geqq 3$ pairs of ESCC tissues, and between the SHEE and SHEEC cell lines as well.

\section{Re-annotation of differential AS events}

AS events used in the previous analysis were annotated with human genome (hg19) alternative events v2.0, and their annotations were derived from Ensembl genes (Ensembl database), knownGenes (UCSC database) and RefSeq genes (NCBI database). In order to further verify the AS event annotations and find out their represented genes and transcripts, AS events with significant differences in at least 3 pairs of ESCC tissue samples were re-annotated. Homo_sapiens.GRCh37.75.gtf from the Ensembl database [25] and GRCh37_latest_genomic. gff from the RefSeq database [26] were chosen for reannotation. Since the mRNA sequences and gene-related annotations of the UCSC database were derived from GenBank and RefSeq databases, those annotations were not adopted for re-annotation of AS events with several criterion described as follows: (i) if three exons in an SE (Skipped exon) event appear in the same transcript, the SE event is judged to be corrected, (ii) if two exons in an RI (Retained intron) event are present in the same transcript and there are no other exons between these two exons, then the RI event annotation is determined to be corrected, (iii) if adjacent exons, for example exons 1-2-4 and 1-3-4, occur in the same transcript in an MXE (Mutually exclusive exon) event, and exons 1-4 do not appear in any one transcript, the MXE event is judged as correct, (iv) if the variable exon (the longer or the shorter exon) and constitutive exons in an A3SS (Alternative 3'splice site) event occurs in the same transcript, and between these two exons there are no other exons, the A3SS event annotation is determined to be corrected, (v) if the variable exon and the constitutive exon in an A5SS (Alternative 5'splice site) appear in the same transcript, and between the two exons there are no other exons, the A5SS event annotation is judged to be correct. Since there are no chromosome numbers in the RefSeq exon 
annotations, the chromosomal locations of exons using RefSeq annotations should be checked later.

\section{Functional enrichment analysis}

DAVID online tools [27] were utilized to perform functional enrichment analysis towards GO biological process and KEGG pathways. The significant cut-off was set to $P<0.05$. Dot plots from $\mathrm{R}$ package named ggplot2 [28] were then applied to visualize the significantly enriched GO terms and KEGG pathways.

\section{Differential AS events occurred both in ESCC tissues and SHEE/SHEEC cell lines}

After the results of differential AS events from the SHEE and SHEEC cell lines were filtered, the following information, including AS event names, $\Psi$ values from the SHEE cell line, $\Psi$ values from the SHEEC cell line and $\Delta \Psi$ values from SHEE/SHEEC cells were selected. The reannotation results of the AS events in ESCC tissues using gene annotations were overlapped with SHEE/SHEEC AS events, then the similar trend of $\Delta \Psi$ values of each overlapped AS event in tissues and in cell lines was judged.

\section{Splicing regulatory network establishment}

Gene expression of each sample was combined and merged with the splicing factor list, which is available from the SpliceAid2 database, which contains 2,220 target sites of 62 human splicing proteins and their expression data in 320 tissues [29]. Spearman correlation tests were performed between splicing factors and the splicing event/genes, by the calculation of the expression levels and the PSI values, accordingly. The significant pairs of splicing factors and splicing genes were chosen to establish a splicing regulatory network. Cytoscape software was used to visualize the splicing regulatory network, which is an open source platform for visualization of molecular interaction [30].

\section{Survival analysis}

The expression and clinical data of 87 ESCC patients was obtained from the Xena browser database (https:// xenabrowser.net/datapages/). The log-rank (Mantel-Cox) test was used to perform survival analysis and a KaplanMeier curve was plotted using GraphPad software.

\section{Statistical analysis}

Statistical analyses were performed using SPSS 19.0 (IBM, Chicago, IL, USA) or R 3.15.3 (Auckland, New Zealand) for Windows. Comparisons of the relative expression of between paired tumor and non-tumor tissues, were performed using a paired $t$-test. Overall survival time was calculated by the Kaplan-Meier method and analyzed by the log-rank test. A two-tailed $P$-value $<0.05$ was considered to be statistical significant.

\section{Results}

Overall mapping rate after genome alignments

Reads from the RNA-seq of each esophageal clinical case were mapped to human genome sequences. The results are shown in Table 1, where the total alignment number of the sequenced fragments ranged from 15 to 39 million, and the comparison rate ranged between 83 and $92 \%$, suggesting the high reliability of these RNA-seq data.

\section{Changes in AS patterns between ESCC tissue/matched normal tissue and two cell lines}

From the results of splicing analysis, Using MISO, a total of 45,439 AS events were detected. Of these, 6,019 AS events were significantly differently spliced in ESCC tissues as compared to normal tissues, with a cut-off of $\mathrm{BF}>5,|\Delta \Psi|>0.2$. These significant AS events included a number of inclusion and exclusion reads $>10$, accounting for $13.25 \%$ of the total number of AS events, suggesting a relative high splicing index in ESCC (Fig. 1a). After filtering out the AS events with inconsistent $\Delta \Psi$ trends in the paired samples, there were 5,150 differential splicing events, including 2,324 exon skipping (SE) events, 1,014 intron retention (RI) events, and 693 mutually exclusive exon (MXE) events, 592 alternative 3' splice site (A3SS) events, and 527 alternative 5' splice site (A5SS) events. The AS results of ESCC tissue samples showed that the proportion of SE events that could be detected were the most abundant at about $33.6 \%$, RI events at $6.9 \%$, MXE 6.8\%, A3SS 8.5\%, and A5SS 6.4\% of the total events. The percentage of SE events in differential splicing events was still the highest as $4.8 \%$, while RI events comprising 2.5\%, MXE events comprising 1.5\%, A3SS events comprising 1.2\%, and A5SS events comprising approximately $1.1 \%$. Individual differences were detected during MISO analysis which were more obvious in the differential splicing events (Fig. 1b). From the volcano plot, it could be observed that the $\Delta \Psi$ values and $\log 2(\mathrm{BF})$ values in detectable AS events and the differentially spliced events were symmetrically distributed (Fig. 1c), Also, it could be seen from the and bar plot that the $\Delta \Psi$ trend did not have a significant difference between AS event type (Fig. 1d).s

On the other hand, a total of 32,891 AS events were detected in the SHEE/SHEEC cell lines, of which a total of 928 AS events were significantly differentially spliced, accounting for approximately $2.8 \%$ of the total detectable AS events (Fig. 1e). The threshold for judging the differences in AS events in the SHEE/SHEEC cell lines was the same as applied in ESCC AS event filtration. In the detectable AS events, the proportion of SE was the 
Table 1 Summary of TopHat2 genomic alignments

\begin{tabular}{|c|c|c|c|c|c|c|}
\hline \multirow[t]{2}{*}{ Samples } & \multicolumn{2}{|l|}{ Total reads } & \multicolumn{2}{|c|}{ Mapped reads } & \multicolumn{2}{|l|}{ Overall reads } \\
\hline & Left & Right & Left & Right & Mapping_rate (\%) & Aligned_pairs \\
\hline $\mathrm{B} 782 \mathrm{~N}$ & $51,906,891$ & $51,906,891$ & $45,391,832$ & $42,285,862$ & 84.50 & $39,419,636$ \\
\hline B782T & $20,187,921$ & $20,187,921$ & $18,022,068$ & $16,864,424$ & 86.40 & $15,851,609$ \\
\hline $\mathrm{B} 783 \mathrm{~N}$ & $22,772,025$ & $22,772,025$ & $20,876,811$ & $20,292,461$ & 90.40 & $19,220,668$ \\
\hline B783T & $22,026,613$ & $22,026,613$ & $20,199,656$ & $19,665,894$ & 90.50 & $18,669,500$ \\
\hline $\mathrm{B} 785 \mathrm{~N}$ & $23,881,681$ & $23,881,681$ & $21,879,991$ & $20,946,206$ & 89.70 & $19,806,614$ \\
\hline B785T & $27,545,958$ & $27,545,958$ & $25,310,820$ & $24,617,731$ & 90.60 & $23,314,980$ \\
\hline $\mathrm{B} 786 \mathrm{~N}$ & $19,526,756$ & $19,526,756$ & $18,043,943$ & $17,643,909$ & 91.40 & $16,752,687$ \\
\hline B786T & $20,424,033$ & $20,424,033$ & $18,841,925$ & $18,319,670$ & 91.00 & $17,453,969$ \\
\hline B788N & $22,166,170$ & $22,166,170$ & $20,319,140$ & $19,346,438$ & 89.50 & $18,276,699$ \\
\hline B788T & $19,329,401$ & $19,329,401$ & $17,949,865$ & $17,468,161$ & 91.60 & $16,651,551$ \\
\hline $\mathrm{B} 791 \mathrm{~N}$ & $25,487,339$ & $25,487,339$ & $22,803,788$ & $22,091,751$ & 88.10 & $20,629,163$ \\
\hline B791T & $19,730,469$ & $19,730,469$ & $17,587,161$ & $16,688,030$ & 86.90 & $15,713,877$ \\
\hline B794N & $21,773,037$ & $21,773,037$ & $20,089,962$ & $18,134,197$ & 87.80 & $17,197,455$ \\
\hline B794T & $27,630,784$ & $27,630,784$ & $24,796,070$ & $22,364,260$ & 85.30 & $20,946,236$ \\
\hline B797N & $24,393,756$ & $24,393,756$ & $22,517,747$ & $21,882,580$ & 91.00 & $20,713,525$ \\
\hline B797T & $20,082,035$ & $20,082,035$ & $18,745,753$ & $18,269,290$ & 92.20 & $17,451,339$ \\
\hline B798N & $32,153,939$ & $32,153,939$ & $29,786,198$ & $26,930,352$ & 88.20 & $25,572,896$ \\
\hline B798T & $32,468,547$ & $32,468,547$ & $30,246,172$ & $27,414,170$ & 88.80 & $26,154,479$ \\
\hline B799N & $25,194,910$ & $25,194,910$ & $23,448,172$ & $21,151,887$ & 88.50 & $20,095,525$ \\
\hline B799T & $20,897,751$ & $20,897,751$ & $19,481,307$ & $17,526,890$ & 88.50 & $16,701,290$ \\
\hline $\mathrm{B} 800 \mathrm{~N}$ & $26,328,835$ & $26,328,835$ & $24,474,467$ & $22,048,959$ & 88.40 & $20,927,261$ \\
\hline B800T & $24,186,510$ & $24,186,510$ & $22,481,444$ & $20,362,666$ & 88.60 & $19,317,914$ \\
\hline $\mathrm{B} 801 \mathrm{~N}$ & $22,799,054$ & $22,799,054$ & $21,390,618$ & $19,363,520$ & 89.40 & $18,518,368$ \\
\hline B801T & $26,946,581$ & $26,946,581$ & $25,259,555$ & $22,713,737$ & 89.00 & $21,717,932$ \\
\hline $\mathrm{B} 804 \mathrm{~N}$ & $27,121,722$ & $27,121,722$ & $25,391,238$ & $22,801,422$ & 88.80 & $21,774,130$ \\
\hline B804T & $24,985,226$ & $24,985,226$ & $23,383,899$ & $21,149,641$ & 89.10 & $20,212,413$ \\
\hline C199N & $29,776,640$ & $29,776,640$ & $26,261,213$ & $25,254,804$ & 86.50 & $23,366,546$ \\
\hline С199T & $35,651,673$ & $35,651,673$ & $30,502,787$ & $29,013,594$ & 83.50 & $26,708,316$ \\
\hline $\mathrm{C} 200 \mathrm{~N}$ & $21,494,047$ & $21,494,047$ & $19,784,129$ & $19,242,503$ & 90.80 & $18,270,154$ \\
\hline C200T & $19,588,861$ & $19,588,861$ & $17,953,109$ & $17,510,470$ & 90.50 & $16,566,068$ \\
\hline SHEE & $55,085,029$ & $55,085,029$ & $47,868,214$ & $44,812,830$ & 84.1 & $41,691,361$ \\
\hline SHEEC & $54,582,526$ & $54,582,526$ & $47,769,458$ & $44,796,267$ & 84.8 & $42,325,127$ \\
\hline
\end{tabular}

largest, at 55.9\%, with RI at 9.2\%, MXE at $10.9 \%$, A3SS accounting for $13.6 \%$, and A5SS at $10.3 \%$. Of the differential splicing events, the number of SE, RI, MXE, A3SS, and A5SS events were 455, 131, 152, 96, and 94, accounting for $49 \%, 14.1 \%, 16.4 \%, 10.3 \%$, and $10.1 \%$ of the total number of differential splicing events, separately (Fig. 1f). The distribution of both total and differential AS is similar in ESCC tissues and ESCC cell lines.

\section{Re-annotation of AS events in paired ESCC tissues}

To identify the genes and transcripts representing the differential AS events and to filter out incorrect AS events, as described in the methods, we re-annotated all differentially splicing events found in at least 3 paired samples. The number of filtered significant AS events changed across the different categories of AS due to the re-annotation, but the event trend had minimal changes (Fig. 2a). For SE events, there were a total of 222 and 151 significant alternatively spliced events before and after re-annotation, reducing the number of AS events to approximately $68 \%$ of all events. There were a total of 134 RI significant AS events before reannotation, and 115 after re-annotation, with a reduction of $14.2 \%$. A total of 62 significant MXE events were identified before re-annotation and only 6 remained after re-annotation, reducing the total number to $9.7 \%$. 57 significant A3SS events were found before re-annotation while 42 remained after re-annotation. 35 out 
of 52, 67.3\%, significant A5SS events were identified after the re-annotation. Among these above results, SE events are the most prevalent in ESCC. The number of MXE events was reduced the most, because the two exons that should have been mutually exclusive in the MISO official MXE event annotation were observed to exist in the same transcript. Thus, a total of 349 significant AS events including five types were identified after re-annotation in 15 matched pairs of ESCC and normal tissue samples, of which transcripts/genes representing 169 AS events could be found both in the Ensembl and RefSeq annotations, whereas 114 could only be identified in the RefSeq annotations, and 66 AS events only in the Ensembl annotations (Fig. 2b). Through we found different annotations from these two popular databases, the majority of our annotation was found in both databases. We have also calculated the fractions of AS events representing protein-coding mRNA and ncRNA (non-coding RNA) (Fig. 2c). The percentage for ncRNA ranged from $10 \%$ to more than $20 \%$ in total events, suggesting alternative splicing is prevalent for ncRNA and its critical role in cancer [31]. The top 10 most prevalent splicing events that occurred in the paired samples are shown in Table 2. To better illustrate the different splicing patterns, HNRNPC, CALD1, KIAA1217, TPM1, VCL and ZNF207 were selected from the top genes and their splicing patterns are visualized by Sashimi plots (Fig. 3). These included representative examples for MXE, A5SS, RI and SE splicing types, which indicates the splicing diversity in ESCC.

\section{Overlapping AS events in ESCC tissues and SHEE/SHEEC cell lines}

A total of 37 significant AS events were identified in both ESCC tissues and cell lines, 15 of which showed the same trend of $\Delta \Psi$ values. Among these 15 AS events, there were $4 \mathrm{SE}$ events, 7 RI events, 2 MXE events, and 2 A5SS events (Additional file 1: Table S1). Though the overlapping rate is quite low, we presume this might reflect the consensus and difference between clinical samples and cultured cell lines.

\section{Classification of AS events according to representative transcript types}

After the re-annotation of the 349 AS events from ESCC tissues, 312 events represented at least one protein-coding transcript, which accounted for $89.4 \%$ of the total AS events. The remaining 37 AS events did not represent any protein-coding transcripts, including protein coding transcripts, lncRNAs, and microRNAs (Additional file 2: Table S2). Among the 235 AS events re-annotated by Ensembl transcript annotations, 200 events represented at least one proteincoding transcript, which accounted for $85.1 \%$ of the total AS events, and the remaining 35 AS events represented non-protein-coding transcripts. Of the 283 variable splicing events re-annotated by RefSeq transcript annotations, 266 events represented at least one protein-coding transcript, which accounted for $94 \%$ of the total AS events, and the remaining 17 AS events represented non-protein-coding transcripts. These results suggest that splicing of ncRNA transcripts could be just as prevalent as protein-coding transcripts.

\section{Functional enrichment revealed the potential roles of AS transcripts}

To identify the biological processes and pathways affected by splicing abnormalities in ESCC, we performed the enrichment analyses. A total of 270 protein-coding genes were selected for functional and pathway enrichment analysis from the results of alternative splicing events after re-annotation of differential AS events with gene annotations from the Ensembl and RefSeq databases. A total of 234 genes were enriched in 543 biological processes. There were 382 biological processes satisfying the threshold of $P<0.05$, and 39 genes were enriched in 12 pathways, of which 10 pathways satisfied $P<0.05$. Interestingly, there were 4 KEGG pathways and 20 biological processes that potentially related to invasion and metastasis of cancer cells, such as adherens junction, ECM-receptor interaction, focal adhesion, regulation of cell mobility, regulation of cell migration and cell-matrix adhesion

\footnotetext{
(See figure on next page.)

Fig. 1 Distribution of RNA splicing events in ESCC after MISO analysis. a Percentages of differentially-spliced AS events with significance in total AS events found in ESCC clinical samples compared to the matched normal samples. $\mathbf{b}$ Plot depicts the fraction of events detected for each AS type (blue) compared to events with significantly different expression in ESCC tissues compared to matched normal tissues (red). c Scatter plot of all the AS events identified using MISO. The X-axis represents $\Delta \Psi$ values, and the Y-axis represents log2 (BF) values. The shape of the dots indicates the type of AS event. Specifically, a hollow square indicates an SE event; cross indicates an RI event; solid square indicates an MXE event; triangle indicates an A5SS event; and circle indicates an A3SS event. Alternative splicing events with BF $>5$ and $\Delta \Psi>0.2$ are colored in blue. $\mathbf{d}$ Relative fraction of each AS event affected positively or negatively by ESCC. e Percentage of alternative splicing events showing significant changes in SHEE/SHEEC cell line association compared to detectable alternative splicing events showing no difference. $\mathbf{f}$ Plot depicts the fraction of events detected for each AS type (blue) compared to events with significantly different expressions in SHEE/SHEEC cell lines (red)
} 


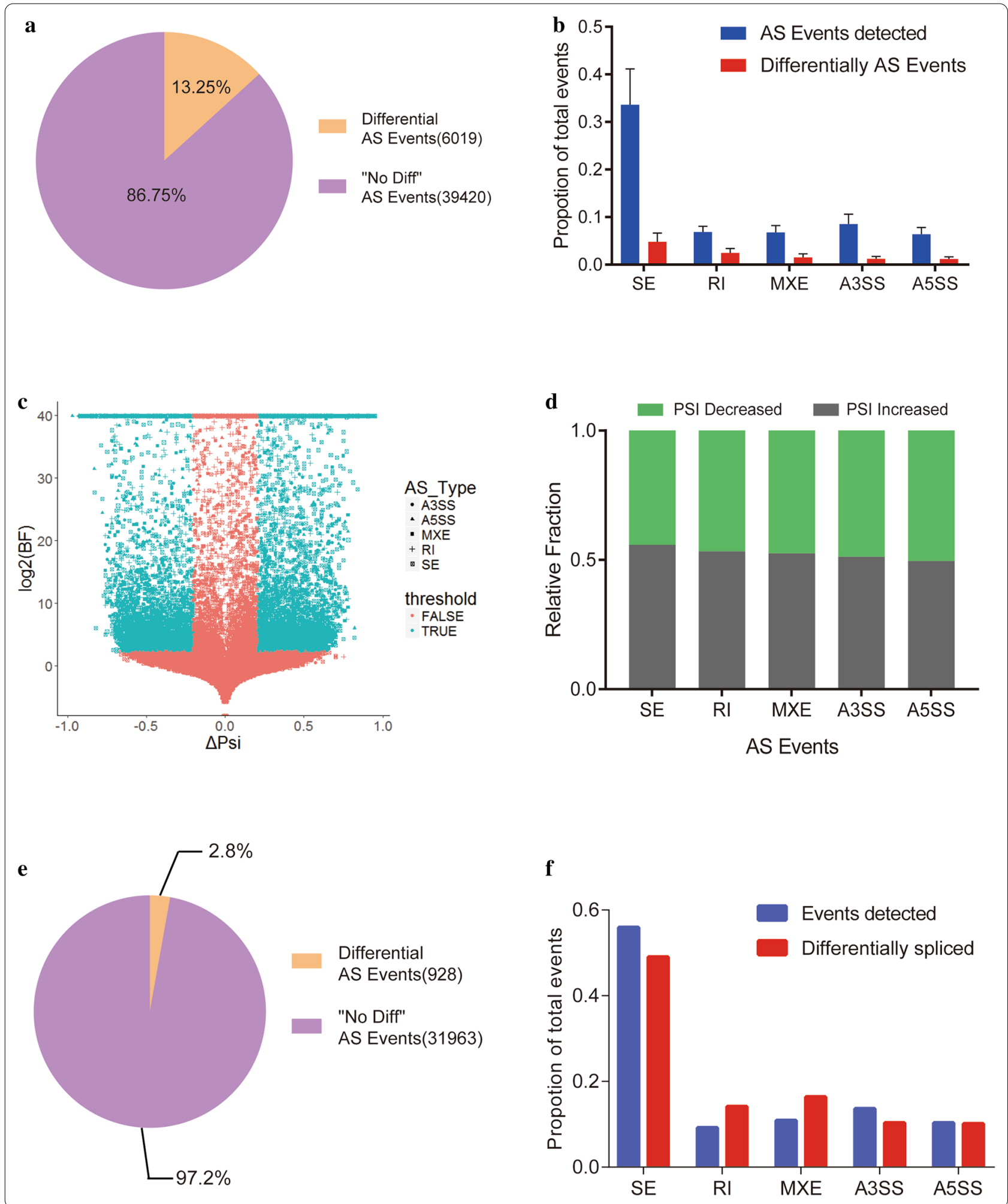

(Fig. 4). These results indicated that AS in ESCC might involve multiple cellular functions to promote tumor progression.

\section{Splicing regulatory network in ESCC}

To find the regulators of the splicing alterations in ESCC, we constructed a splicing regulatory network by 

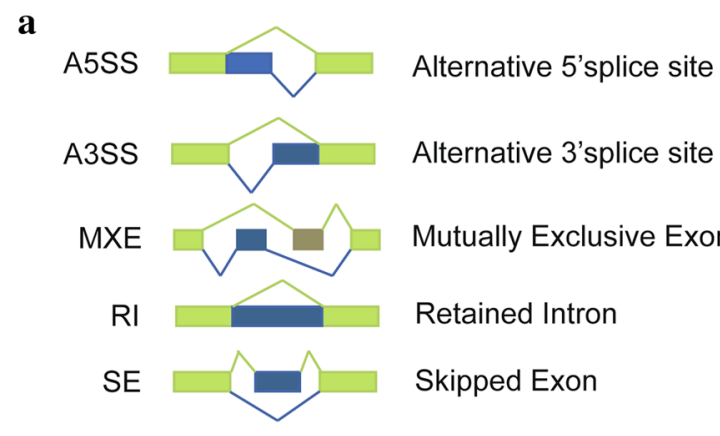

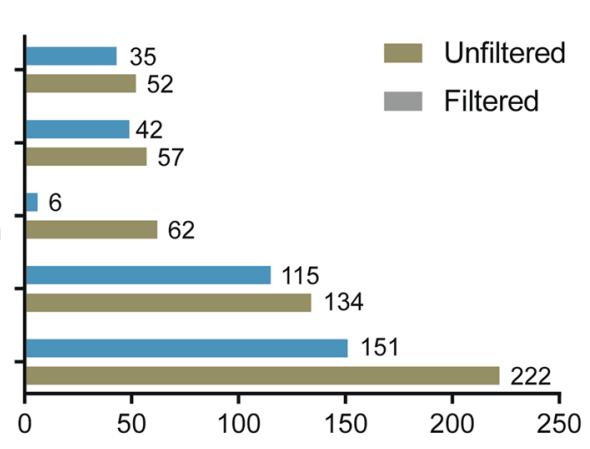

c
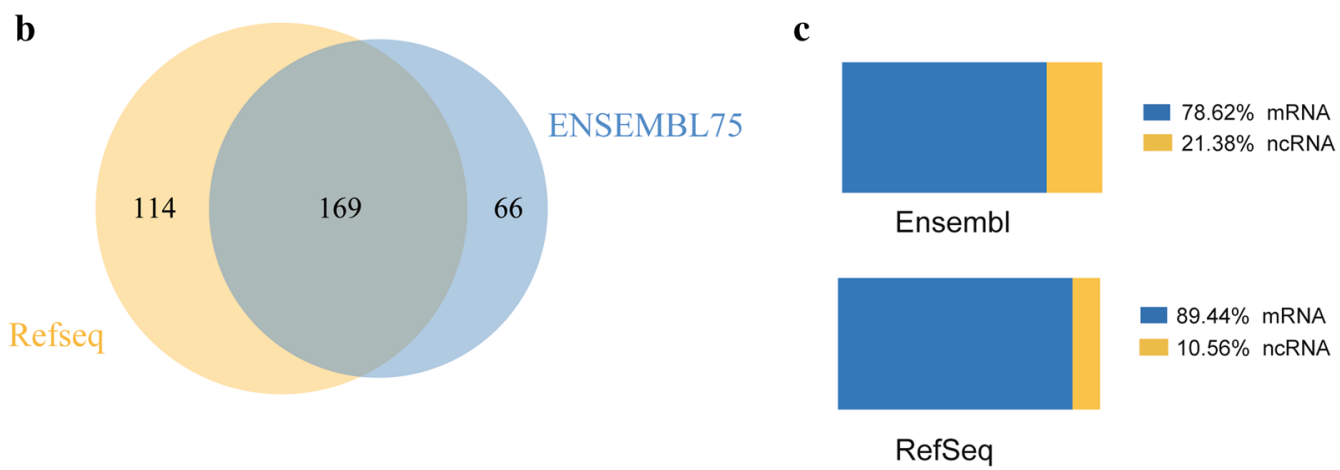

Fig. 2 Distribution of RNA splicing events with significant differences in ESCC after re-annotation with Ensembl and RefSeq gene annotations, a Plot in the left panel indicates exon models of the types of AS assessed by MISO analysis. In the right panel, the different types of AS events before and after re-annotation were quantified with transcript annotations from Ensembl and RefSeq database, respectively. b A total of 283 and 235 splicing events with significantly altered PSI values were identified using transcript annotations from the RefSeq and Ensembl databases, respectively. In total, 169 splicing events were identified in both database transcript annotations. c Percentage of protein coding transcripts (blue) and non-coding transcripts (yellow) representing AS events after re-annotation analysis

calculating the FPKM values of splicing factors and PSI values of AS events. Filtered with a cut-off of $P<0.05$ after Spearman correlation analysis, 5,999 splice factorsplicing event pairs and 5,511 splice factor-target gene pairs were chosen to build a splicing regulatory network. This network involved 81 splicing factors and 223 differential AS events (Fig. 5). Most of splicing factors could regulate dozens of target genes. On the other hand, one target gene could be processed by several splicing factors, suggesting the co-operation between these factors. Next, Splicing factors that were differentially expressed in
ESCC and matched normal tissues were measured in two public microarray datasets (GSE53624 and GSE23400) and the RNA-seq dataset generated from our laboratory [16]. From these analyses, the splicing factor SF3B4 was significantly differentially expressed in ESCC with a 1.5 fold-change in three different datasets (Table 3).

For the splicing factors in the network, SF3B4 (splicing factor $3 \mathrm{~b}$ subunit 4 ) caused us great interest. The splicing regulatory network for SF3B4 (splicing factor 3b subunit 4) and its target genes (with significant Spearman correlation) were also illustrated, which contained 92 target

(See figure on next page.)

Fig. 3 Sashimi plot of representative splicing changes in ESCC. Diagrams on the left show the read coverage of exons. Plots on the right show the $\psi$ values that occurred at least in two pair ESCC tissues or cell lines, as indicated by different colors. The AS model of this region is represented in each down panel. a-f AS model is shown for HNRNPC, CALD1, KIAA1217, TPM1, VCL and ZNF207, respectively. The curve indicates the splicing sites and the number in the curve suggests RNA-seq reads in this region 


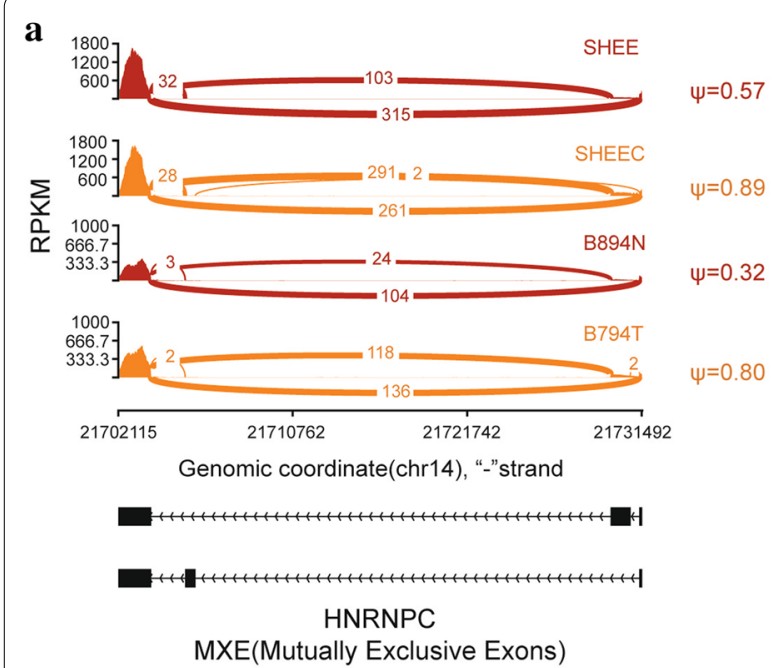

c
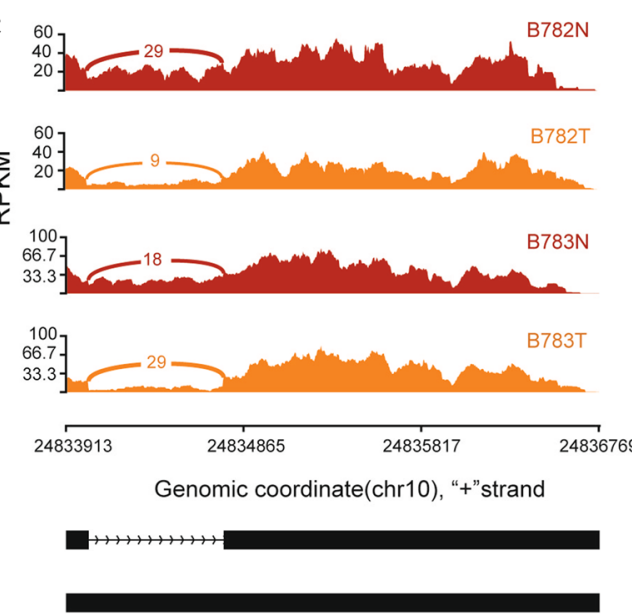

KIAA1217

RI(Retained Intron)

e
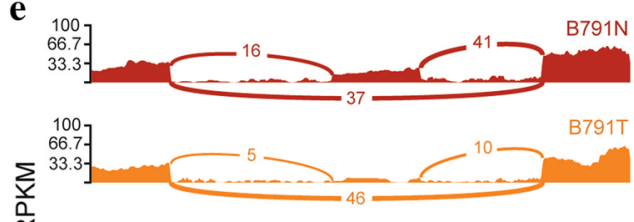

$\frac{1}{\alpha}$

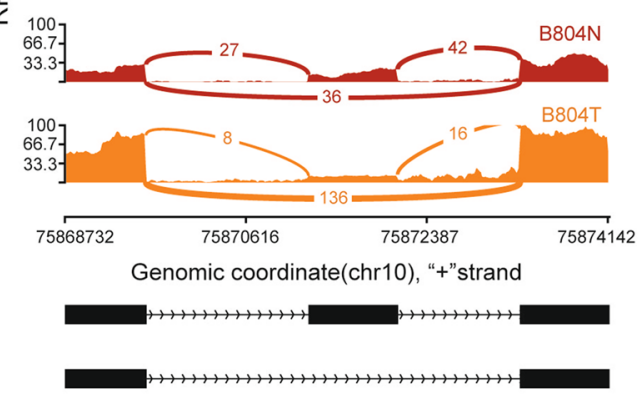

VCL

SE(Skipped Exon)

$\psi=0.07$ b
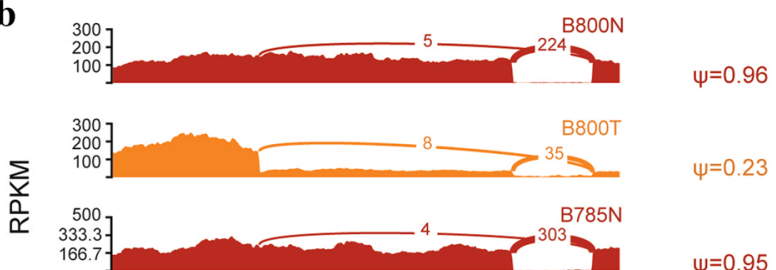

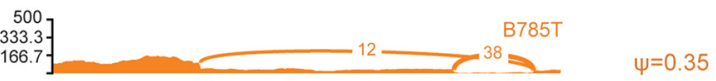

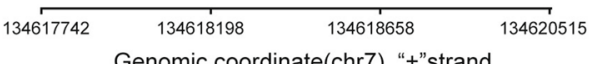

Genomic coordinate(chr7), "+"strand

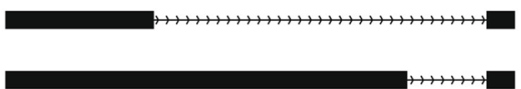

CALD1

A5SS(Alternative 5' splicing site)
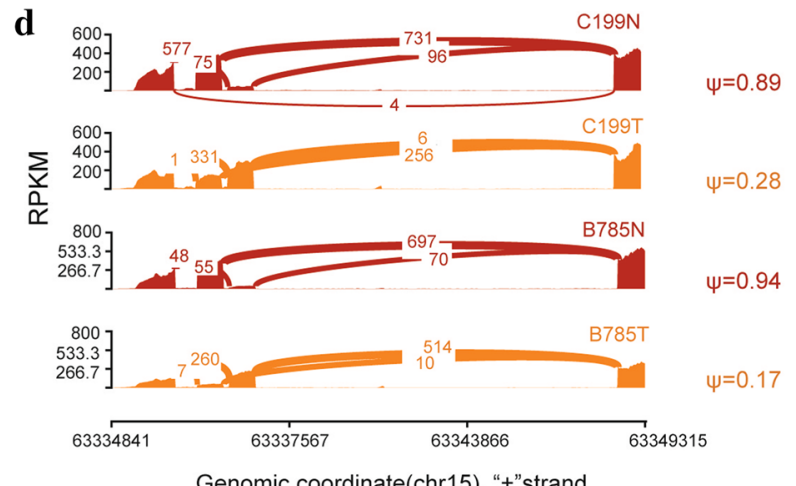

Genomic coordinate(chr15), "+"strand

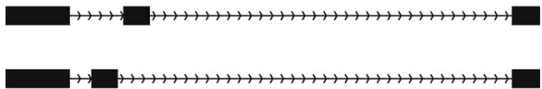

TPM1

MXE(Mutually Exclusive Exons)

f
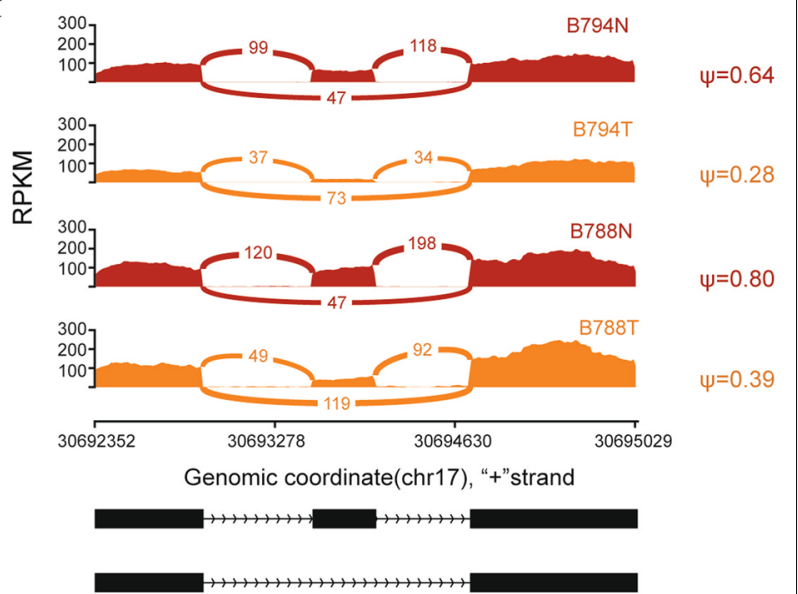

ZNF207

SE(Skipped Exon) 
Table 2 Summary of top 10 alternative splicing events in paired ESCC tissues

\begin{tabular}{|c|c|c|c|}
\hline Splicing event & Gene name & $\begin{array}{l}\text { Count of paired } \\
\text { sample }\end{array}$ & Type \\
\hline chr1:150,483,352:150,483,674:+@chr1:150,483,933:150,484,307:+@chr1:150,484,828:150,485,048:+ & ECM1 & 14 & SE \\
\hline chr10:24,833,910-24,834,032:+@chr10:24,834,756-24,836,772:+ & KIAA1217 & 13 & $\mathrm{Rl}$ \\
\hline chr9:117,835,882:117,836,145:-@chr9:117,808,689:117,808,961:-@chr9:117,804,498:117,804,620:- & TNC & 12 & SE \\
\hline $\begin{array}{l}\text { chr15:63,334,838:63,335,142:+@ @chr15:63,335,905:63,336,030:+@chr15:63,336,226:63,336,351:+ @ } \\
\text { chr15:63,349,184:63,349,317:+ }\end{array}$ & TPM1 & 11 & MXE \\
\hline chr2:238,289,558:238,290,142:-@chr2:238,287,279:238,287,878:-@chr2:238,285,415:238,285,987:- & COL6A3 & 11 & SE \\
\hline chr22:31,495,731:31,495,882:+@echr22:31,496,871:31,497,035:+@chr22:31,500,302:31,500,610:+ & SMTN & 10 & SE \\
\hline chr16:15,808,766:15,808,938:-@chr16:15,802,660:15,802,698:-@chr16:15,796,992:15,797,980:- & MYH11 & 9 & SE \\
\hline chr17:30,692,349:30,692,506: + @chr17:30,693,684:30,693,776: + @chr17:30,694,791:30,695,033: + & ZNF207 & 9 & SE \\
\hline chr7:134,617,739:134,618,141|134,618,828:+@chr7:134,620,439:134,620,516:+ & CALD1 & 9 & A5SS \\
\hline chr9:117,835,882:117,836,145:-@chr9:117,819,432:117,819,704:-@chr9:117,808,689:117,808,961:- & TNC & 9 & SE \\
\hline
\end{tabular}

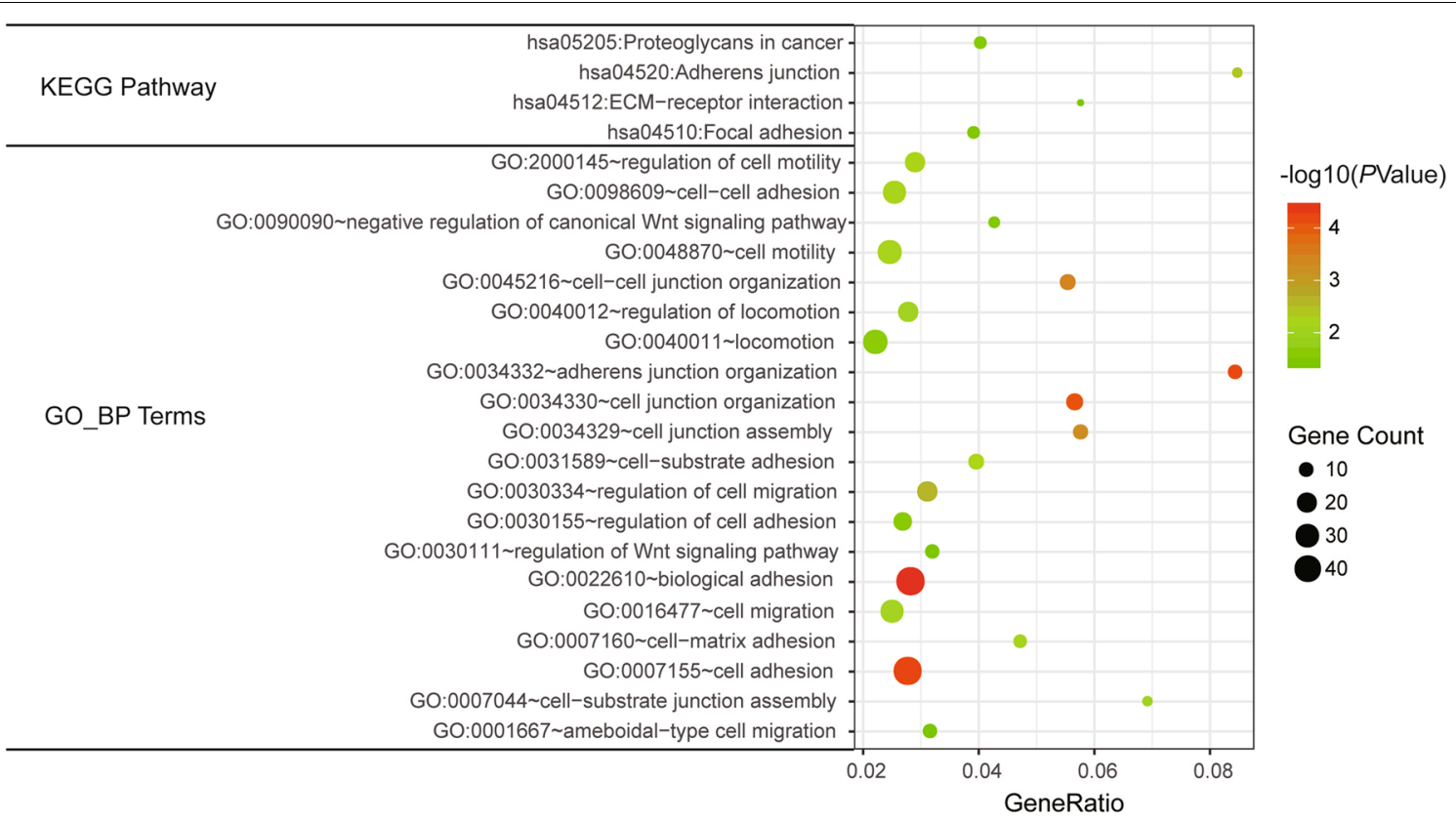

Fig. 4 Dot plot of significant GO_BP terms and KEGG pathways using functional enrichment analyses with aberrant splicing event-related genes

Table 3 Summary of SF3B4 expression using three ESCC dataset

\begin{tabular}{llllll}
\hline Dataset & Number of Samples & FoldChange & Pvalue & Qvalue & R packages \\
\hline RNA-seq & 15 pairs & 1.42 & 0.197 & 0.458 & DESeq \\
GSE53624 & 119 pairs & 1.64 & 0 & 0 & Siggenes \\
GSE23400 & 53 pairs & 1.50 & 0 & 0 & Siggenes \\
\hline
\end{tabular}

genes and 102 abnormal AS events (Fig. 6a), as some genes generated more than one AS event. Moreover, SF3B4 was found to be a survival-related gene in ESCC. In TCGA dataset, ESCC patients with a lower expression level of SF3B4 had a longer survival time (Fig. 6b). The PSI value of SRSF5's RI event was found to have the smallest $p$-value with Spearman correlation analysis with the FPKM of SF3B4 (Fig. 6c). To understand the potential 


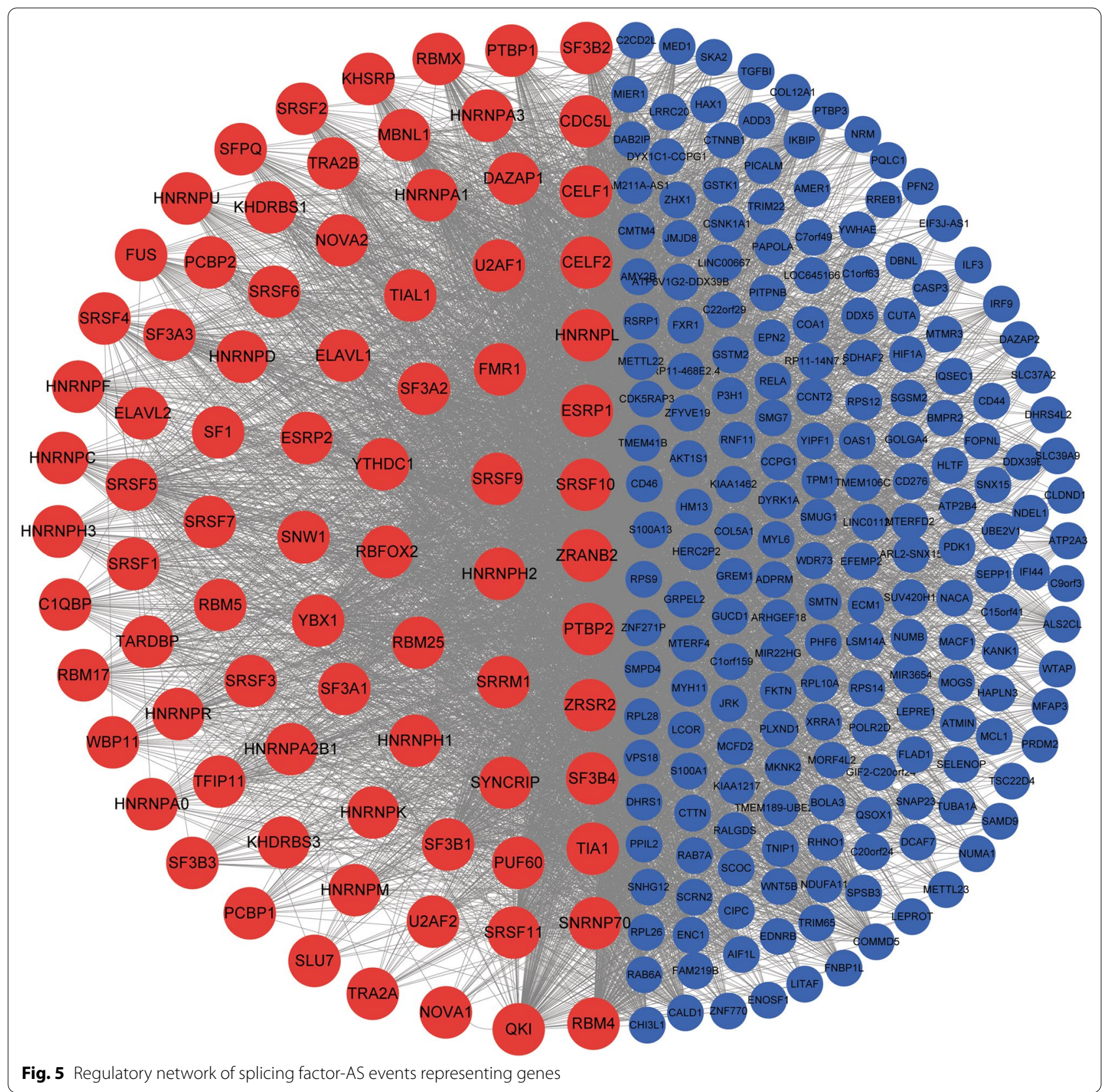

functions of SF3B4, functional enrichment analysis was also performed to identify the biological processes for SF3B4's target genes, resulting in a dot plot of 9 significant biological processes, which involves cell-cell adhesion, NF-KB signaling and regulation of translation (Fig. 6d). Many evidences indicated AS are often linked to the occurrence of cancer driver mutations in genes encoding either core components or regulators of the splicing machinery [32]. However, we searched cBioPortal database (http://www.cbioportal.org/), but no mutation for SF3B4 in current TCGA ESCC clinical samples was found (data not shown).

\section{SF3B4 affects AS after its knockdown}

To validate the regulatory role of SF3B4 on AS events in tumor samples, we have downloaded two RNA-seq datasets with SF3B4 knockdown, and calculated the inclusive levels of AS events with IncLevelDifference between control shRNA and SF3B4 knockdown (methods described in Additional file 3). From the 102 SF3B4 regulated AS events described above. We selected 36 AS events with 

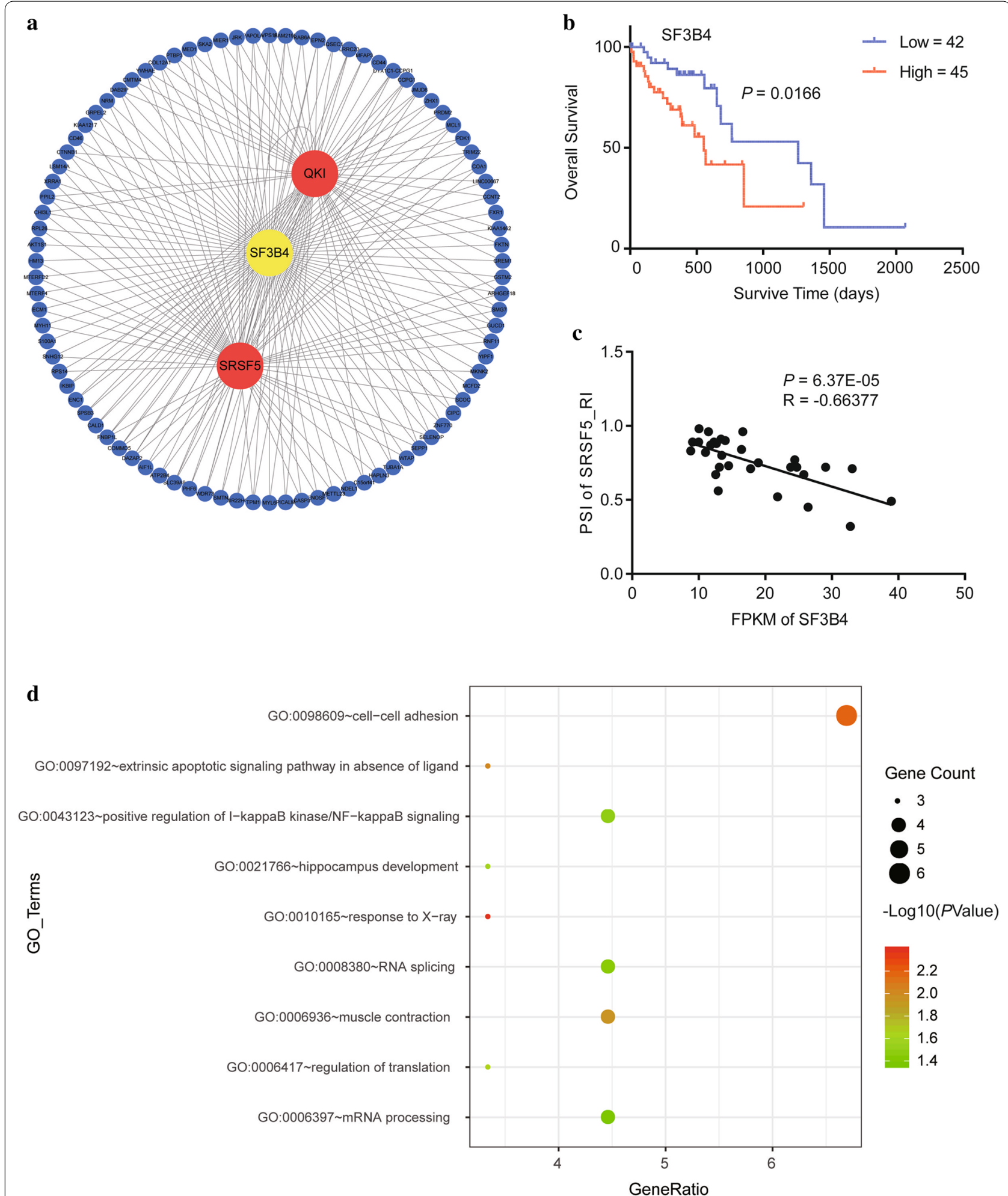

Fig. 6 SF3B4-related regulatory network in ESCC. a SF3B4 splicing regulatory network. b K-M curve for SF3B4 in ESCC. c Scatter plot indicating correlation between SF3B4 expression and PSI value of the RI event of SRSF5. d Dot plot of GO_BP terms using alternatively-spliced SF3B4-targeted genes 
top Spearman test P-value and top correlation $(1>\mid$ Rho $\mid$ $>0.5$ ), and named them as the top SF3B4 regulated AS events in ESCC samples. Subsequently, we tried to sieve out the overlapping results between the top SF3B4 regulated AS events in ESCC and significant AS events $(F D R<0.05)$ in either of the two validation datasets. Six overlapping AS events regulated by SF3B4 in tumor samples were finally verified, including $1 \mathrm{SE}$ event (ATP2B4) and 5 RI events in 4 genes (SRSF5, PHF6, SNHG12, KIAA1217). It is interesting to point out that their Rho values are all negative, indicating SF3B4 represses intron retention in these transcripts. These results also suggest that SF3B4 could regulate AS in a pan-cancer manner, including in ESCC. The detailed information of the top 36 SF3B4 regulated AS events in ESCC are provided in Additional file 4: Table S3, with the 6 verified AS events indicated in the validated cell lines.

\section{Discussion}

As an important step in post-transcriptional regulation, AS greatly contributes to proteome diversity, as well as cancer progression and development. As a common phenomenon in cancers, it has been reported that an endogenous soluble form of VEGFR-2, a product of alternative splicing, is present in humans and mice [33]. Our previous research found that endogenous soluble VEGFR-2 is down-regulated in ESCC, but its high expression is an independent prognostic factor for poor survival [33]. We identified a novel splice variant of LOXL2 (Lysyl oxidase-like 2), named LOXL2 $\Delta 72$, which lacks 72 nucleotides encoding 24 amino acids [34]. Though LOXL2 $\Delta 72$ has dramatically reduced enzymatic activity, it promotes greater cell migration and invasion than its wild type counterpart [34]. However, alternative splicing studies in ESCC remain at a low-throughput level, and analyses of AS events in ESCC on a genome-wide scale are still rare. Although aberrant splicing events in ESCA samples have been found and related to overall survival, detailed splicing patterns of ESCC are still unknown, and splicing-mode changes between ESCC and matched normal tissues have not been characterized [35]. In this paper, previously published next-generation sequencing data was used to study AS in ESCC. Because our RNAseq data was generated from ESCC tissue and matched normal tissue of the same patient, our data is suitable for MISO analyses and splicing-mode change characterization. There are RNA-seq data generated from a pair of cell line samples, an immortalized human esophageal epithelial cell line (SHEE) and its malignant transformed cell line (SHEEC), which was also used as a validating dataset. This comparison reflects the consistence and difference between cancer tissues and cultured cells while considering their AS pattern.
In total, 6,019 significant AS events were identified in 15 pairs of ESCC tissues. It is also noteworthy that a large proportion of the AS events were found to only have significant differences in a small number of paired samples, which suggests that AS differences in many genes might have a similar impact on the development of ESCC. The proportion of AS events with $\Delta \Psi>0$ and $\Delta \Psi<0$ in ESCC tends to be consistent, which indicates that the current AS classification system cannot promise all the true AS types, and the real AS modes may be more complicated. We have noticed that the AS annotations in MISO are based on existing transcripts, so alternative splicing analysis can not discover new transcripts, but unknown AS regulatory mechanisms based on known genes and transcripts could be revealed by the MISO analysis. Even so, there are many problems with the official MISO AS annotations, in which several exons in mutually exclusive exon events are found present in the same transcript, making the accuracy of the annotation confusing. In this study, transcriptional annotations from Ensembl and RefSeq database were used to re-annotate the previously analyzed AS events and to remove incorrect splicing events to make the subsequent analysis more accurate.

Many laboratories have reported some clues on the mechanism of alternative splicing affecting cancers. For example, the normal transcript for CD44 was expressed in normal gastric tissue, and the upregulation of its splice variant is associated with invasion and metastasis of gastric tumors [36]. Higher expression of CD44 variant 6 has been reported in gastric tumor with lymph node metastasis, suggesting that CD44 variant 6 plays a role in the metastasis of gastric cancer [37]. BP1, a splicing isoform of DLX4, is highly expressed in endometrial cancer, which correlates with patient poor prognosis, cancer pathological grade, tumor invasion and metastasis [38]. For the first time, from the perspective of systems biology, we show the biological processes and KEGG pathways affected by alternative splicing in ESCC development. By functional enrichment analyses, multiple GO terms and pathways are observed in relation to cell junction and cell migration, indicating that altered splicing may promote invasion and metastasis in ESCC.

For the top differential AS genes generated from ESCC in this study, many of them have been reported to play an important role in tumor progression. HNRNPC is a typical splicing factor, and its regulation by alternative splicing has not been reported. Alternative splicing of ZNF207 has been reported to be regulated by SRSF11 [39], but its AS pattern has not been reported. VCL encodes an F-actin-binding cytoskeletal protein, compared to VCL-001, VCL-201 contains an extra exon named exon 19. The skipped exon 19 is reported to be increasingly preferred in colon tumor cells compared to 
normal mucosa [40], which is consistent with its Sashimi plot model shown in this study. CALD1 is a calmodulin- and actin-binding protein with regulatory functions, and has been reported to play an important role in cell movement. In colon cancer tissues, the longest CALD1 transcript is reported to be reduced, with the increase of its shorter splicing isoform [40]. However, Sashimi plot results in this study show that there is an alternative $5^{\prime}$ splice site event in CALD1, which also involves a relatively down-regulated expression of its longest isoform. Intron retention of KIAA1217 has been found in nonsmall cell lung cancer, but RT-PCR results do not support this result [41]. Our results indicate that there is an RI event for KIAA1217, but the retained intron tends to be included in matched normal tissues. Regarding TPM1, it has been reported that exon 6 of TPM1 has two types, TPM1-6A and TPM1-6B, but are the same 76bp size. In bladder cancer and prostate cancer, TPM1 exon 6A and $6 \mathrm{~B}$ are reported to have opposite splicing trends [42]. We also show there is an MXE event in TPM1s, and both the mutually exclusive exons are 76bp long. Though our top AS genes are generated from paired ESCC tissues and cell lines, they need further verification, both in clinical samples and cultured cell lines.

Splicing factors, which participate in the ribonucleoprotein (RNP) complexes that play key roles in AS regulation often drive different AS patterns in a tissue- and cell-type-specific manner, both in normal and cancerous conditions [43]. A key role for SF3B4 in diseases in regards to its splicing function has been revealed in recent years. A heterozygosity for SF3B4 mutations is found in Rodriguez syndrome, which leads to reduced SF3B4 synthesis and defects in mRNA splicing, primarily exon skipping [44]. Overexpressing SF3B4 resulted in a mis-splicing of Kruppel-like factor 4 (KLF4), a tumor suppressor-encoding gene, into a non-functional transcript in cancer cells, and thus promoting tumorigenesis in HCC [45].

But the relationship between SF3B4 and ESCC has not been identified. In this paper, we found that SF3B4 is up-regulated in ESCC, which is similar to the results of an HCC study, in which SF3B4 is an up-regulated diagnostic marker [46]. Moreover, according to the log-rank (Mantel-Cox) test, SF3B4 can be identified as a prognostic marker of ESCC. Consistently, a new paper found over-expression of SF3B4 may play a crucial role in the lymphatic progression of ESCC, during the submission of our study [47]. Spearman correlation analysis was utilized to calculate the correlation between the degree of splicing events and the expressions of splicing factors in ESCC, according to a previous method [48]. SF3B4 was also found to play an important role in regulating 92 protein coding genes and their aberrant splicing events in ESCC. Functional enrichment analysis indicates that SF3B4's downstream targets may play a role in cell-cell junction and nuclear factor $\mathrm{kB}(\mathrm{NF}-\mathrm{kB})$ processes.

To identify the target genes of SF3B4 in tumors, significant AS events were investigated in SF3B4 knockdown cells. Six AS events regulated by SF3B4 in tumor samples overlapped with the top SF3B4 regulated AS events in ESCC. The oncogenic role of SRSF5 [49], PHF6 [50], SNHG12 [51] and KIAA1217 [52] have been reported in different tumors, but we firstly discovered that intron retention in these genes are repressed by SF3B4. Retained intron (RI) repression potentially leads to productive transcripts, because retained introns usually produce premature termination codons, which may generate unproductive, unstable transcripts or truncated proteins [53]. These results reveal that SF3B4 regulates the expression pattern of many cancer-associated genes through AS mechanisms. However, it is still necessary to verify their regulatory mechanism and subsequent biological effects through validation experiments.

\section{Conclusions}

In this study, AS events were analyzed from RNA-seq data from matched normal and ESCC tissue samples pairs and two cell lines where $13.25 \%$ significant differential AS events were identified, which are involved in cell junction and migration. The splicing factor SF3B4 is a potential prognostic factor and a splicing regulator in ESCC. In summary, we identified thousands of significant differential AS events which might be related to the development of ESCC.

\section{Supplementary Information}

The online version contains supplementary material available at https://doi. org/10.1186/s13578-021-00546-z.

Additional file 1: Table S1. Summary of alternative splicing events with significant difference in ESCC tissues \& cell lines.

Additional file 2: Table S2. Alternative splicing events representing noncoding RNA.

Additional file 3. Materials and methods.

Additional file 4: Table S3. SF3B4 regulated alternative splicing events tested in SF3B4 shRNA knockdown cell lines.

\section{Acknowledgements}

We thank Dr. Stanley Lin from Shantou university for helpful suggestions. We are grateful to Dr. Deanna Tiek from Northwestern University for proof-reading the manuscript.

\section{Authors' contributions}

Conceptualization, LYX, EML and BLW; Funding acquisition, LYX, EML and BLW; Data curation, JYD and CQL; Software and Methodology, JYD, CQL, YWC, ZPD, QYW, ZDT and CS; Project administration, QXX, WJB, LL and WL; Writing-original draft, JYD; Writing - review and editing, LYX, EML and BLW. All authors read and approved the final manuscript. 


\section{Funding}

This work was supported in part by the National Science Foundation of China (Grant Nos. 81672473, 8150213861602292, 81772532 and 81872372), National Cohort of Oesophageal Cancer of China (Grant No. 2016YFC09014000), and the Science and Technology Program of Guangdong (No.2014A030310390, No.2017A030313181).

\section{Availability of data and materials}

The datasets used and/or analyzed during the current study are available from the corresponding author on reasonable request.

\section{Ethics approval and consent to participate} Not applicable.

\section{Consent for publication}

Not applicable.

\section{Competing interests}

The authors declare that they have no conflicts of interest.

\section{Author details}

1 Key Laboratory of Molecular Biology for High Cancer Incidence Coastal Chaoshan Area, Shantou University Medical College, Shantou 515041, China. 2 Department of Biochemistry and Molecular Biology, Shantou University Medical College, Shantou 515041, China. ${ }^{3}$ School of Medical Informatics, Harbin Medical University, Daqing Campus, Daqing 163319, China. ${ }^{4}$ Department of Pathology, Shantou Central Hospital, Affiliated Shantou Hospital of Sun Yat-sen University, Shantou 515041, China. ${ }^{5}$ Institute of Oncologic Pathology, Shantou University Medical College, Shantou 515041, China.

Received: 27 June 2020 Accepted: 29 January 2021

Published online: 09 February 2021

\section{References}

1. Smyth EC, Lagergren J, Fitzgerald RC, Lordick F, Shah MA, Lagergren P, Cunningham D. Oesophageal cancer. Nat Rev Dis Primers. 2017;3:17048.

2. Siegel RL, Miller KD. Cancer statistics. CA. 2019;69(1):7-34.

3. Zlotorynski E, Splicing. Going in circles. Nat Rev Genet. 2018;19(2):64-5.

4. Kastner B, Will CL, Stark H, Lührmann R. Structural Insights into Nuclear pre-mRNA Splicing in Higher Eukaryotes. Cold Spring Harb Perspect Biol. 2019;11(11):a032417.

5. Eymin B. Targeting the spliceosome machinery: a new therapeutic axis in cancer? Biochem Pharmacol. 2020;1:114039.

6. Zhang $P$, Zhang XO, Jiang T, Cai L, Huang X, Liu Q, Li D, Lu A, Liu Y, Xue W, Zhang P, Weng Z. Comprehensive identification of alternative back-splicing in human tissue transcriptomes. Nucleic Acids Res. 2020;48(4):1779-89.

7. Sciarrillo R, Wojtuszkiewicz A, Assaraf YG, Jansen G, Kaspers GJL, Giovannetti $\mathrm{E}$, Cloos J. The role of alternative splicing in cancer: From oncogenesis to drug resistance. Drug Resist Updat. 2020;53:100728.

8. Scotti MM, Swanson MS. RNA mis-splicing in disease. Nat Rev Genet. 2016;17(1):19-32.

9. Bonnal SC, López-Oreja I, Valcárcel J. Roles and mechanisms of alternative splicing in cancer - implications for care. Nat Rev Clin Oncol. 2020;17(8):457-74.

10. Rahman MA, Krainer AR, Abdel-Wahab O. SnapShot splicing alterations in cancer. Cell. 2020;180(1):208-8.e1.

11. Kahles A, Lehmann KV, Toussaint NC, Hüser M, Stark SG, Sachsenberg T, Stegle O, Kohlbacher O, Sander C. Cancer genome Atlas Research Network, Rätsch G. Comprehensive analysis of alternative splicing across tumors from 8,705 patients. Cancer Cell. 2018;34(2):211-24.e6.

12. Lian H, Wang A, Shen Y, Wang Q, Zhou Z, Zhang R, Li K, Liu C, Jia H. Identification of novel alternative splicing isoform biomarkers and their association with overall survival in colorectal cancer. BMC Gastroenterol. 2020;20(1):171.

13. Qu Y, Chen Y, Zhang L, Tian L. Construction of prognostic predictor by comprehensive analyzing alternative splicing events for colon adenocarcinoma. World J Surg Oncol. 2020;18(1):236.
14. Song J, Liu YD, Su J, Yuan D, Sun F, Zhu J. Systematic analysis of alternative splicing signature unveils prognostic predictor for kidney renal clear cell carcinoma. J Cell Physiol. 2019;234(12):22753-64.

15. Katz Y, Wang ET, Airoldi EM, Burge CB. Analysis and design of RNA sequencing experiments for identifying isoform regulation. Nat Methods. 2010;7(12):1009-15.

16. Li CQ, Huang GW, Wu ZY, Xu YJ, Li XC, Xue YJ, Zhu Y, Zhao JM, Li M, Zhang J, Wu JY, Lei F, Wang QY, Li S, Zheng CP, Ai B, Tang ZD, Feng CC, Liao LD, Wang SH, Shen JH, Liu YJ, Bai XF, He JZ, Cao HH, Wu BL, Wang MR, Lin DC, Koeffler HP, Wang LD, Li X, Li EM, Xu LY. Integrative analyses of transcriptome sequencing identify novel functional IncRNAs in esophageal squamous cell carcinoma. Oncogenesis. 2017;6(2):e297.

17. Andrews S. FastQC: A quality control tool for high throughput sequence data. http://www.bioinformatics.babraham.ac.uk/projects/fastqc/.

18. Lee CM, Barber GP, Casper J, Clawson H, Diekhans M, Gonzalez JN, Hinrichs AS, Lee BT, Nassar LR, Powell CC, Raney BJ, Rosenbloom KR, Schmelter D, Speir ML, Zweig AS, Haussler D, Haeussler M, Kuhn RM, Kent WJ. UCSC Genome Browser enters 20th year. Nucleic Acids Res. 2020;48(D1):D756-61

19. Kim D, Pertea G, Trapnell C, Pimentel H, Kelley R, Salzberg SL. TopHat2: accurate alignment of transcriptomes in the presence of insertions, deletions and gene fusions. Genome Biol. 2013;14(4):R36.

20. Langmead B, Salzberg SL. Fast gapped-read alignment with Bowtie 2. Nat Methods. 2012;9(4):357-9.

21. Trapnell C, Williams BA, Pertea G, Mortazavi A, Kwan G, van Baren MJ, Salzberg SL, Wold BJ, Pachter L. Transcript assembly and quantification by RNA-Seq reveals unannotated transcripts and isoform switching during cell differentiation. Nat Biotechnol. 2010;28(5):511-5.

22. Anders $\mathrm{S}$, Huber W. Differential expression analysis for sequence count data. Genome Biol. 2010;11(10):R106.

23. Li J, Chen Z, Tian L, Zhou C, He MY, Gao Y, Wang S, Zhou F, Shi S, Feng X, Sun N, Liu Z, Skogerboe G, Dong J, Yao R, Zhao Y, Sun J, Zhang B, Yu Y, Shi X, Luo M, Shao K, Li N, Qiu B, Tan F, Chen R, He J. LncRNA profile study reveals a three-IncRNA signature associated with the survival of patients with oesophageal squamous cell carcinoma. Gut. 2014;63(11):1700-10.

24. Su H, Hu N, Yang HH, Wang C, Takikita M, Wang QH, Giffen C, Clifford R, Hewitt SM, Shou JZ, Goldstein AM, Lee MP, Taylor PR. Global gene expression profiling and validation in esophageal squamous cell carcinoma and its association with clinical phenotypes. Clin Cancer Res. 2011;17(9):2955-66.

25. Aken BL, Ayling S, Barrell D, Clarke L, Curwen V, Fairley S, Fernandez Banet J, Billis K, García Girón C, Hourlier T, Howe K, Kähäri A, Kokocinski F, Martin FJ, Murphy DN, Nag R, Ruffier M, Schuster M, Tang YA, Vogel JH, White S, Zadissa A, Flicek P, Searle SM. The Ensembl gene annotation system. Database. 2016;2016:baw093.

26. Haft DH, DiCuccio M, Badretdin A, Brover V, Chetvernin V, O’Neill K, Li W, Chitsaz F, Derbyshire MK, Gonzales NR, Gwadz M, Lu F, Marchler GH, Song JS, Thanki N, Yamashita RA, Zheng C, Thibaud-Nissen F, Geer LY, MarchlerBauer A, Pruitt KD. RefSeq: an update on prokaryotic genome annotation and curation. Nucleic Acids Res. 2018;46(D1):D851-60.

27. Jiao X, Sherman BT, Huang da W, Stephens R, Baseler MW, Lane HC, Lempicki RA. DAVID-WS: a stateful web service to facilitate gene/protein list analysis. Bioinformatics. 2012;28(13):1805-6.

28. Wickham H. ggplot2: Elegant Graphics for Data Analysis. New York: Springer-Verlag; 2016

29. Piva F, Giulietti M, Burini AB, Principato G. SpliceAid 2: a database of human splicing factors expression data and RNA target motifs. Hum Mutat. 2012;33(1):81-5

30. Smoot ME, Ono K, Ruscheinski J, Wang PL, Ideker T. Cytoscape 2.8: new features for data integration and network visualization. Bioinformatics. 2011;27(3):431-2.

31. Yuan JH, Liu XN, Wang TT, Pan W, Tao QF, Zhou WP, Wang F, Sun SH. The MBNL3 splicing factor promotes hepatocellular carcinoma by increasing PXN expression through the alternative splicing of IncRNA-PXN-AS1. Nat Cell Biol. 2017;19(7):820-32.

32. Bonnal SC, López-Oreja I, Valcárcel J. Roles and mechanisms of alternative splicing in cancer - implications for care. Nat Rev Clin Oncol. 2020;17(8):457-74.

33. Wu ZY, Chen T, Zhao Q, Huang JH, Chen JX, Zheng CP, Xu XE, Wu JY, Xu $L Y, L i$ EM. Altered expression of endogenous soluble vascular endothelial 
growth factor receptor- 2 is involved in the progression of esophageal squamous cell carcinoma. J Histochem Cytochem. 2013 May;61 (5):340-7.

34. Zou HY, Lv GQ, Dai LH, Zhan XH, Jiao JW, Liao LD, Zhou TM, Li CQ, Wu BL, Xu LY, Li EM. A truncated splice variant of human lysyl oxidase-like 2 promotes migration and invasion in esophageal squamous cell carcinoma. Int J Biochem Cell Biol. 2016;75:85-98.

35. Mao S, Li Y, Lu Z, Che Y, Sun S, Huang J, Lei Y, Wang X, Liu C, Zheng S, Zang R, Li N, Li J, Sun N, He J. Survival-associated alternative splicing signatures in esophageal carcinoma. Carcinogenesis. 2019;40(1):121-30.

36. Li Y, Yuan Y. Alternative RNA splicing and gastric cancer. Mutat Res. 2017;773:263-73

37. Chen XY, Wang ZC, Li H, Cheng XX, Sun Y, Wang XW, Wu ML, Liu J. Nuclear translocations of beta-catenin and TCF4 in gastric cancers correlate with lymph node metastasis but probably not with CD44 expression. Human pathology. 2005;36(12):1294-301.

38. Zhang L, Wan Y, Jiang Y, Zhang Z, Shu S, Cheng W, Lang J. Overexpression of BP1, an isoform of Homeobox Gene DLX4, promotes cell proliferation, migration and predicts poor prognosis in endometrial cancer. Gene. 2019;707:216-23.

39. Toh CX, Chan JW, Chong ZS, Wang HF, Guo HC, Satapathy S, Ma D, Goh GY, Khattar E, Yang L, Tergaonkar V, Chang YT, Collins JJ, Daley GQ, Wee KB, Farran CA, Li H, Lim YP, Bard FA, Loh YH. RNAi Reveals Phase-Specific Global Regulators of Human Somatic Cell Reprogramming. Cell Rep. 2016:15(12):2597-607.

40. Bisognin A, Pizzini S, Perilli L, Esposito G, Mocellin S, Nitti D, Zanovello P, Bortoluzzi S, Mandruzzato S. An integrative framework identifies alternative splicing events in colorectal cancer development. Molecular oncology. 2014;8(1):129-41.

41. Langer W, Sohler F, Leder G, Beckmann G, Seidel H, Grone J, Hummel $M$, Sommer A. Exon array analysis using re-defined probe sets results in reliable identification of alternatively spliced genes in non-small cell lung cancer. BMC Genomics. 2010;11:676.

42. Thorsen K, Sørensen KD, Brems-Eskildsen AS, Modin C, Gaustadnes M, Hein AM, Kruhøffer M, Laurberg S, Borre M, Wang K, Brunak S, Krainer AR, Tørring N, Dyrskjøt L, Andersen CL, Orntoft TF. Alternative splicing in colon, bladder, and prostate cancer identified by exon array analysis. Mol Cell Proteomics. 2008;7(7):1214-24.

43. Rahman MA, Nasrin F, Bhattacharjee S, Nandi S. Hallmarks of Splicing Defects in Cancer: Clinical Applications in the Era of Personalized Medicine. Cancers (Basel). 2020;12(6):1381.
44. Marques F, Tenney J, Duran I, Martin J, Nevarez L, Pogue R, Krakow D, Cohn DH, Li B. Altered mRNA Splicing, Chondrocyte Gene Expression and Abnormal Skeletal Development due to SF3B4 Mutations in Rodriguez Acrofacial Dysostosis. PLoS Genet. 2016;12(9):e1006307.

45. Shen Q, Nam SW. SF3B4 as an early-stage diagnostic marker and driver of hepatocellular carcinoma. BMB Rep. 2018;51(2):57-8.

46. Shen Q, Eun JW, Lee K, Kim HS, Yang HD, Kim SY, Lee EK, Kim T, Kang K, Kim S, Min DH, Oh SN, Lee YJ, Moon H, Ro SW, Park WS, Lee JY, Nam SW. Barrier to autointegration factor 1, procollagen-lysine, 2-oxoglutarate 5-dioxygenase 3 , and splicing factor $3 \mathrm{~b}$ subunit 4 as early-stage cancer decision markers and drivers of hepatocellular carcinoma. Hepatology. 2018;67(4):1360-77.

47. Kidogami S, Iguchi T, Sato K, Yoshikawa Y, Hu Q, Nambara S, Komatsu H, Ueda M, Kuroda Y, Masuda T, Mori M, Doki Y, Mimori K. SF3B4 Plays an Oncogenic Role in Esophageal Squamous Cell Carcinoma. Anticancer Res. 2020 May;40(5):2941-6.

48. Richards AL, Watza D, Findley A, Alazizi A, Wen X, Pai AA, Pique-Regi R, Luca F. Environmental perturbations lead to extensive directional shifts in RNA processing. PLoS Genet. 2017;13(10):e1006995.

49. Yang S, Jia R, Bian Z. SRSF5 functions as a novel oncogenic splicing factor and is upregulated by oncogene SRSF3 in oral squamous cell carcinoma. Biochim Biophys Acta Mol Cell Res. 2018;1865(9):1161-72.

50. Yu Q, Yin L, Jian Y, Li P, Zeng W, Zhou J. Downregulation of PHF6 Inhibits Cell Proliferation and Migration in Hepatocellular Carcinoma. Cancer Biother Radiopharm. 2019;34(4):245-51.

51. Shi J, Ding W, Lu H. Identification of long non-coding RNA SNHG family as promising prognostic biomarkers in acute Myeloid Leukemia. Onco Targets Ther. 2020;13:8441-50.

52. Dickinson A, Saraswat M, Mäkitie A, Silén R, Hagström J, Haglund C, Joenväärä S, Silén S. Label-free tissue proteomics can classify oral squamous cell carcinoma from healthy tissue in a stage-specific manner. Oral Oncol. 2018;86:206-15.

53. Jacob AG, Smith CWJ. Intron retention as a component of regulated gene expression programs. Hum Genet. 2017;136(9):1043-57.

\section{Publisher's note}

Springer Nature remains neutral with regard to jurisdictional claims in published maps and institutional affiliations.
Ready to submit your research? Choose BMC and benefit from:

- fast, convenient online submission

- thorough peer review by experienced researchers in your field

- rapid publication on acceptance

- support for research data, including large and complex data types

- gold Open Access which fosters wider collaboration and increased citations

- maximum visibility for your research: over $100 \mathrm{M}$ website views per year

At BMC, research is always in progress.

Learn more biomedcentral.com/submissions 